\section{A map of gene copy numbers}

A sizeable fraction of cases

of developmental delay

in children are caused by

mistakes in the number of

copies of certain genes in their

DNA.

Evan Eichler at the University of Washington in Seattle and his colleagues constructed a large and detailed map of human copy number variations (CNVs), which arise from DNA deletions, duplications or other mutations. The authors used a technique called array comparative genomic hybridization to measure CNVs in 15,767 children with intellectual disabilities and various birth defects.

They estimate that $14.2 \%$ of intellectual disability and developmental delay is caused by CNVs that cover large stretches - 400 kilobases or more - of DNA. They also found 14 disease-causing CNVs that were previously either unknown or only weakly supported by evidence. Nature Genet. http://dx.doi. org/10.1039/ng.909 (2011)

\section{CANCER}

\section{Double hit from small molecules}

A class of small molecules kills cancer cells but leaves normal ones unharmed by targeting two proteins that are dysregulated in almost all cancers.

Katerina Gurova at the Roswell Park Cancer Institute in Buffalo, New York, and her colleagues screened chemical libraries for molecules that both activate the tumoursuppressor p53 and inhibit NF- $\kappa B$, which helps to regulate cell proliferation. They identified a group of molecules, called curaxins, that killed human tumour cells in vitro and inhibited the growth of human tumours in mice.

Unlike current chemotherapeutic drugs, which typically break DNA, curaxins bind to it, changing the architecture of the chromatin - the coiled-up structure in which DNA is packaged. This traps a protein complex called FACT within the chromatin, which shifts the activity of the $\mathrm{p} 53$ and NF- $\kappa \mathrm{B}$ proteins. FACT expression is higher in tumour cells than in normal ones, suggesting that the complex could be a new drug target.

Sci. Transl. Med. 3, 95ra74 (2011)

\section{DEVELOPMENTAL BIOLOGY}

\section{Embryo rhythm predicts success}

In the hours that follow fertilization, the cytoplasm inside mouse eggs moves with a characteristic, although not uniform, rhythm. Screening human embryos for these movements might boost the success rate of in vitro fertilization (IVF).

Magdalena ZernickaGoetz at the University of Cambridge, UK, and her team took rapid time-lapse microscopy footage of mouse eggs for two hours after sperm entry and measured the cells' fluid dynamics. Fertilization induced oscillations in the cells' calcium ion concentration, which, in turn, caused the eggs' protein skeletons to contract rhythmically.

Embryos in which contractions had been more rapid but less frequent were more likely to lead to successful pregnancy in surrogate mice. Nature Commun. 2, 417 (2011) \section{IMMUNOLOGY \\ Prion-like immune trigger}

Prion proteins are responsible for a class of neurodegenerative disorders, including Creutzfeldt-Jakob disease, in which misshapen prions cause other prions to misfold and form fibrous aggregates. Researchers at the University of Texas Southwestern Medical Center

COMMUNITY CHOICE

The most viewed papers in science

\title{
Water + acid + graphene $=$ power
}

\section{HIGHLY READ \\ on pubs.acs.org in July}

water over carbon nanotubes.

Flowing acidic water over a few layers of graphene - atom-thick sheets of carbon - generates ten times the energy previously achieved by running acidic

Water flowing over carbon nanotubes can produce only a small electric current in the direction of the flow. Nikhil Koratkar at Rensselaer Polytechnic Institute in Troy, New York, and his team added hydrochloric acid to water and streamed it over flat sheets of graphene, generating 85 nanowatts of power using a few hundred square micrometres of graphene film.

The group's simulations suggest that the voltage is generated by chloride ions that hop along the surface of graphene, interacting with and dragging the material's electrons along. The authors say that the graphene sheets boosted power generation because of their high electron mobility and continuous surface for efficient ion gliding. Nano Lett. 11, 3123-3127 (2011)

in Dallas have found a human protein that behaves like a disease-causing prion to trigger an antiviral immune response.

Zhijian Chen and his group studied virus-infected human cells and focused on the protein MAVS, which is activated when viral RNA binds to certain cell receptors. They found that, on infection, MAVS molecules form aggregates - similar to those of disease-linked prions - that convert more MAVS to aggregates. This activates MAVS to trigger the antiviral response. The authors suggest that this conversion process serves to amplify this immune response, boosting its sensitivity to viral infection.

Cell 146, 448-461 (2011)

\section{EVOLUTIONARY BIOLOEY}

\section{Why unrelated wasps help}

The sterile workers of social insects such as bees typically sacrifice reproduction to help their genetic relatives. But European paper wasps

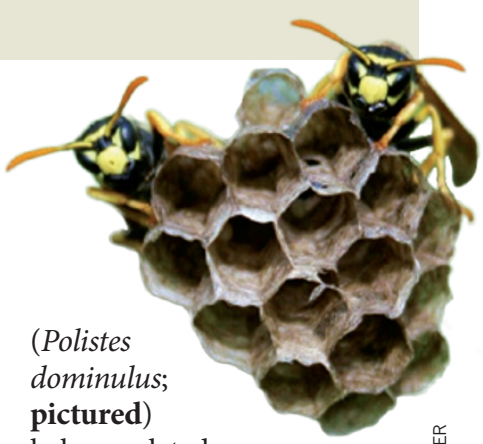

help unrelated

individuals to breed and researchers have discovered why: it affords them the possibility of inheriting the breeder's nest and then producing their own offspring.

Female paper wasps build new nests every spring, either individually or in small groups of females, not all of which are related. Ellouise Leadbeater, now at the Institute of Zoology in London, and her colleagues found that subordinate females produce more offspring than lone breeders; sometimes reproducing while still subordinate, but more often after inheriting the nest and becoming dominant.

Science 333, 874-876 (2011)

\section{$\rightarrow$ NATURE.COM}

For the latest research published by Naturevisit:

www.nature.com/latestresearch 